\title{
Logical problems with Prokasy's assessment of contingency relations in classical skin conductance conditioning
}

\author{
JOHN J. FUREDY, CONSTANTINE X. POULOS, and KARL SCHIFFMAN \\ University of Toronto, Ontario, Canada
}

\begin{abstract}
Prokasy has not dealt with the main issue raised in the Furedy, Poulos, and Schiffman (1975) paper: that "adjustments" to previously random schedules can result in violations of randomness. With regard to those points which Prokasy has chosen to discuss we have argued, first, that the logical basis of Prokasy's method of assessing contingencies is faulty in that it leads to paradoxical consequences. Second, direct quotes are provided to show that, as indicated in our previous paper, and contrary to Prokasy's (1975) claim, doubts concerning the associative status of first-interval electrodermal responses have been published by several investigators.
\end{abstract}

In a previous methodological paper, Furedy, Poulos, and Schiffman (1975) discussed some logical inadequacies of a study by Prokasy, Williams, Kumpfer, Lee, and Jensen (1973), stemming from what Prokasy (1975) has now called "adjustments" to schedules in an attempt to produce functional CS+, CS -, and RS stimuli. Since, in comments in this journal issue, Prokasy (1975) has not dealt with this problem, we refer the reader to the relevant section of our previous paper (Furedy et al., 1975, p. 100), with special reference to the example quoted from Rescorla (1967, p. 79) in which the impropriety of such "adjustments" is clearly illustrated by a hypothetical instance which closely parallels the essential flaw in the Prokasy et al. (1973) procedure.

With respect to those issues which Prokasy (1975) did take up, we would like to comment in terms of the two general issues raised in our previous paper (Furedy et al.. 1975): assessment of the contingency formulation and the associative status of first-interval SCRs.

\section{ASSESSMENT OF THE CONTINGENCY FORMULATIONS IN SCR CONDITIONING: LOGICAL CONSIDERATIONS}

Paradoxical Consequences of the "Overlap" Criterion

In essence, randomness, or zero contingency involves a set of operations such that a signal (i.e., an RS) provides no predictive information about the occurrence of UCS above and beyond predictions based on background cues. On what basis does one make these calculations? Prokasy chooses to base his analysis on the basis of RS and UCS "overlap." However, the mechanical adoption of a simple physical overlap criterion leads to paradoxical consequences. One consequence is that a 1 -sec CS which was always followed by a UCS at a 5-sec interstimulus interval (ISI) in a "trace-conditioning" (e.g., Kimble, 1961) arrangement, would have to be classified by the overlap criterion as a $\mathrm{CS}_{-}$, since sub- jects would never "have experienced an overlap" (Prokasy, 1975, p. 518) of the CS with the UCS. Difficulties with adopting Prokasy's overlap criterion extend beyond the trace-conditioning paradigm. To apply the overlap criterion to another example, consider the case where a 5-sec CS is immediately followed by the UCS, i.e., a "delay" (Kimble, 1961) conditioning paradigm with no physical overlap between CS and UCS. Again, by Prokasy's overlap criterion, this CS would have to be classified not as a CS+ but as a CS-. These difficulties are further compounded by recognizing that the latter example (delay with no overlap) is just the paradigm used by Prokasy et al. (1973) in their study of what they reported to be CS+, RS, and CS - . ' Yet, if Prokasy's (1975) overlap criterion is strictly adopted, a criterinn on which all the computations and tables of his paper rest, it becomes clear that the probability of a US in the presence of a CS+ equals the probability of a US in the presence of a CS-, i.e., both probabilities are zero, since in neither case are there any occurrences of UCS in physical overlap with the CS. Clearly, any criterion for assessing contingency relations which is unable to distinguish between a CSt and a CS- in an SRC paradigm $^{3}$ such as Prokasy's (1975) criterion, is an inadequate basis for computing contingency relations. Accordingly, since the tables and computations provided by Prokasy (1975) are based on what appears to be an untenable overlap criterion, they are not appropriate either to decide the question of whether the Toronto nominal RSs were closer to being CS-s than RSs (as he asserts), or to determine whether the Utah nominal RSs were closer to being CSts than RSs (as we have suggested.

\section{Determining CS-UCS Contingency: The Need for Consistently Applied Criteria \\ Contingency relations can, in fact, be validly compu- ted on the more reasonable assumption that in SCR}


conditioning, CS/UCS associations can be formed even in the absence of CS/UCS overlaps. ${ }^{4}$ In part of our previous analysis of the Utah study, the nominal RS in that study was shown to be highly excitatory on the basis of expected probability ${ }^{5}$ of UCS occurrence within $13 \mathrm{sec}$ of RS onset (Furedy et al., 1975, p. 101). The 13-sec interval was not arbitrarily chosen; rather, it was the interval which Prokasy et al. (1973) explicitly used in order to experimentally produce their desired contingencies. Specifically, as indicated in the Prokasy et al. paper (1973), the adjustments of scheduled UCS occurrence were such that any UCS which would have occurred within $13 \mathrm{sec}$ following the onset of the stimulus to serve as CS- were omitted. If $13 \mathrm{sec}$ is an effective interval for producing negative contingencies, logical consistency demands that the 13-sec interval be used as the basis for all contingency calculations. Thus, it is difficult to justify the reasoning behind using one interval for opperationally producing negative contingencies (i.e., the 13-sec interval) and using another interval (i.e., duration of a 5 -sec CS) for subsequently calculating all contingencies. It should be added that the experimental use of a 13-sec interval seems a reasonable one. In SCR conditioning, ISIs of $10 \mathrm{sec}$ are not uncommon, and $13 \mathrm{sec}$ would seem to encompass the effective predictive interval for the SCR system.

\section{Purpose of Toronto Studies in Relation to Application of Contingency Formulation to SCR Conditioning}

In his analysis of the randomness of the Toronto studies, Prokasy again mainly relies on a physical RSUCS overlap criterion which, as we have shown above, leads to paradoxical consequences in judging contingencies. In addition, Prokasy seems to find the use of a low density rate of UCS occurrence as somehow beyond the boundary conditions for assessing implications of contingency theory in SCR conditions. Thus, he asserts that it is "difficult to understand what it means to base a contingency on an expected one or two overlaps of RS and UCS" (p. 518).

In this regard, it is useful to reiterate brietly the reason for the particular UCS density rate used in the Toronto studies (cf. Furedy et al., 1975, P. 99), Footnote 1). As indicated, the intent was to assess the random procedure as a control for common automatic con. ditioning studies.

This is obviously important for interpreting published studies and for designing new ones. Prokasy's suggestion that contingencies may not show their effect unless there is a high density of UCS may be valid. However, the suggestion cannot logically serve as a criticism of the design of the Toronto studies. which were explicitly intended to examine the operation of contingencies under the lower-UCS-density conditions which hold in the typical SCR conditioning study.

\section{ASSOCIATIVE STATUS OF FIRST-INTERVAL SCRs}

The other point made by Prokasy which bears some comment is his claim that Furedy et al. (1975) created an "erroneous impression" that other investigators have recently denied associative study status to the FIR (Prokasy, 1975, p. 520). Moreover, Prokasy also asserts that, contrary to Furedy et al. (1975), not a single investigator has ever suggested that analogies based on skeletal (e.g., eyeblink) response systems should determine CR specification in SCR conditioning. Prokasy's assertions are inaccurate. For example, a widely cited paper by Stewart, Stern, Winokur, and Fredman (1961, p. 66) concluded that one can use "criteria for defining true conditioned CSR in terms of the response latency, following methods used by previous investigators working with eyeblink and startle conditioning." Again, and more recently, it has been claimed that: Many investigators of GSR conditioning differentiate between short latency responses, which presumably do not reflect the classical conditioning response" (Suboski, Brace, Jarrold, Teller, \& Dieter, 1972, p. 415). We also find it puzzling that Prokasy (1975) cited the review of Dengerink and Taylor (1971) to support his view that "the associative status of the FIR has not been in serious doubt for at least a decade" (p. 520), since these investigators appear at the very least ambivalent on the question. For example, these reviewers asserted that while the FIR is merely an OR, SIRs "are adaptive ... (serving) ... the adaptive function of allowing the organism to prevent signaled injury to the skin" (Dengerink \& Taylor, 1971, p. 358). In addition, they stated in their abstract that, while FIRs uniquely decrease over trials, later-occurring responses "show a negatively accelerated growth curve over trials" (Dengerink \& Taylor, 1971, p. 358), i.e., only nonFlRs show the conventional "learning" function. Finally, there are the comments of Prokasy and Ebel (1967) that "there is a striking parallel between some of our first-response results and the alpha response in eyelid conditioning. Alpha responses decrease over trials" (Prokasy \& Ebel, 1967, p. 255), a point which, as noted above, Dengerink and Taylor seem to have accepted in their abstract as a unique property of SCR FIRs as distinct from SCR SIRs.

In our earlier, more general paper, we cited all these investigators, but did not provide extensive quotations, believing that it was sufficiently clear that these authors intended to question the associative status of FIRs. Prokasy (1975) apparently feels that we have misread that intent. The above sample of quotes should allow the reader to decide for himself.

\section{REFERENCES}

Denzerink, J. A., \& Taylor, S. P. Multiple responses with differentid properties in delayed galvanic skin response conditioning: A review. Psychophysiology, 1971, 8, 348-360.

Furedy.J.J., Poulos, C. X., \& Schiffman, K. Contingency theary 
and classical autonomic excitatory and inhibitory conditioning: Some problems of assessment and interpretation. Psy chphysiology, 1975, 12, 98-105.

Kimble, G. A. Hilgard and Marquis' conditioning and learning. New York: Appleton-Century-Crofts, 1961

Prokasy, W. F. Random control procedures in classical skin conductance conditioning. Behavior Research Methods \& Instrumentation, $1975,7,516-520$

Prokasy, W. F., \& Ebel, H. C. Three components of the classically conditioned GSR in human subjects. Journal of Experimental Psychology, 1967, 73, 247-256.

Prokasy, W. F., Williams, W. C. Kumpfer, K. I., Lee, W. Y., \& Jensen, W. R. Differential SCR conditioning with two control baselines: Random signal and signal absent. Psychophysiology, 1973.10.145-153

Rescorla, R. A. Pavlovian conditioning and its proper control procedures. Psychological Review, 1967, 74, 71-80

Rescorla, R. A. Conditioned innibition of fear. In W. K. Honig and N. J. Mackintosh (Eds.). Fundamental issues in associative learning. Halifax. Dalhousie University Press, 1969. Pp. 65-89.

Stewart, M. A., Stern, J. A., Winokur, G., \& Fredman, S. An analysis of GSR conditioning. Psychological Review, 1961, 68, 65-89.

Suboski, M. D., Brace, T. G., Jarrold, L. A., Teller, K., \& Dieter, $R$. Interstimulus interval and time estimation in ratings of signaled shock avoidance. Journal of Experimental Psychology, $1972,96,407.415$.

\section{NOTES}

1. We refer the reader to the following samples of text from Prokasy's (1975) paper on this issue: " $\mathrm{CS}-$-' will refer to a sig- for which there is no overlap of signal and UCS" (p. 516) "subjects not receiving a chance RS-UCS overlap have, for all practical purposes, received a CS-, not an RS" (p. 518); "given random assignment of the UCS within the time interval containing RS, one can calculate the expected proportion of time that the $\dot{R} S$ and RCS failed to occupy a common time unit throughout an entire session" (p. 518).

2. The inadequacy of the simple overlap criterion is perhaps also indicated by the fact that, while Prokasy purports to use physical overlap for the basis of his analysis, calculations, and tables, he blurs the criterion by elassifying his own paradigm of delay and no overlap as a case of physical overlap.

3. It should be noted that when the duration of the signal approximates the effective conditioning interval and the CS overlaps the UCS (as is commonly used in assessment of contingency theory in the CER paradigm; cf. Rescorla, 1969), the overlap criterion is acceptable.

4. In our previous paper (Furedy et al., 1975), we used the terminology of UCS occurrence during the presence of a signal intercnangeably with the phrase "closely following Rs onset." Our usage clearly indicated psychological presence in the sense of effective associative interval rather than physical overlop.

5. Expected rather than actual probabilities were used both for expository considerations and because Prokasy et al. (1973 p. 148) used the SCR data from only the first half of their experiment for which probabilities were not calculable from the Prokasy et al. paper.
(Received for publication July 2,1975 ; accepted A ugust 8, 1975.) 